\title{
Annealing behavior of hydrogen-implanted magnetic garnet
}

\author{
C. H. Wilts, H. Awano, ${ }^{\text {a) }}$ and V.S. Speriosu \\ California Institute of Technology, Pasadena, California 91125
}

(Received 5 July 1984; accepted for publication 6 November 1984)

Ferromagnetic resonance spectra and $\mathrm{x}$-ray rocking curves were used to measure magnetic and strain profiles of $\mathrm{Gd}, \mathrm{Tm}, \mathrm{Ga}$ substituted yttrium iron garnet films implanted with $\mathrm{H}_{2}{ }^{+}$at 120 $\mathrm{keV}$ and at doses in the range $(3-80) \times 10^{15}$ ions $/ \mathrm{cm}^{2}$. The maximum strain occurred at a depth of $3600 \AA$, reaching a value of $2.9 \%$ at $40 \times 10^{15}$ ions $/ \mathrm{cm}^{2}$. At the highest dose, the garnet was amorphous in that region which had highest strain at lower dose. The strain has two components, one due to damage and one due to the presence of the hydrogen atoms. The second component disappears for annealing temperatures above $400^{\circ} \mathrm{C}$, at which temperature the hydrogen has been reported to be largely desorbed. The reduction of the first component with annealing follows the same pattern as other implant elements. The magnetic anisotropy exhibits a large anomalous nonlinear increase with dose. The excess over other implantation elements disappears for annealing temperatures above $400^{\circ} \mathrm{C}$. There is no significant change in gyromagnetic ratio with dose or annealing temperatures up to $600^{\circ} \mathrm{C}$.

\section{INTRODUCTION}

The major structural changes created by ion implantation in magnetic garnet are the coherent expansion or strain of the unit cell in the direction perpendicular to the surface and the incoherent displacements of atoms away from ideal crystal sites described by a probability distribution with standard deviation $U .^{1,2}$ The strain increases with dose and has a magnitude of $2.5-3.5 \%$ at the amorphous threshold. $\mathrm{X}$-ray diffraction has established that the relationship between the strain (measured with high precision) and $U$ (measured with low precision) is linear, but the proportionality constant increases with the mass of the implanted species. ${ }^{2}$ For species ranging from $\mathrm{Ne}$ to $\mathrm{He}$ the strain correlates well with calculated nuclear energy deposition density, indicating that it is due to damage rather than to the presence of the implanted dopant. ${ }^{3,4}$ For $\mathrm{Ne}, \mathrm{He}$, and low-dose $\mathrm{H}$ implantation, annealing in air reduces the strain with little change in the shape of the strain profile. ${ }^{2.5}$ After annealing at $1100^{\circ} \mathrm{C}$ the strain due to $\mathrm{Ne}$ implantation is reduced to zero regardless of its initial value.

Implantation with hydrogen at higher doses in the range $10^{16}-10^{17}$ ions $/ \mathrm{cm}^{2}$ produces strain profiles with features different from those obtained with lower doses of hydrogen or the much lower doses of $\mathrm{Ne}$ or He implantation. For high-dose $\mathrm{H}$ implanatation, the strain does not scale with nuclear energy deposition and can be changed significantly by annealing at low temperatures below $300^{\circ} \mathrm{C} .{ }^{4}$ Since hydrogen is known to desorb at low annealing temperatures, ${ }^{6}$ this suggests that at high doses there may be a component of the strain associated with the presence of hydrogen. One aim of the present work was to study in detail the annealing behavior of strain profiles obtained with highdose hydrogen implantation.

Detailed studies of strain and magnetic anisotropy profiles have established that for implantation elements ranging from $\mathrm{He}$ to $\mathrm{Ne}$, the change in magnetic anisotropy depends primarily on the change in local strain, with a linear relation for strain up to $0.5 \%$. This relationship holds equally well near the surface of the film where the density of implanted

a) Now at Sony Corporation, Tokyo, Japan. ions is very small and at the region of mean range where the density of ions is relatively much larger. ${ }^{2,5}$ With increasing annealing temperature, the strain progressively decreases, and the magnetic anisotropy follows the strain closely, except at very high temperatures. Even beyond the linear range the relationship is essentially the same function for both neon and helium implantation and is unaffected by subsequent annealing. In the nonlinear region, the anisotropy shows a progressive saturation beyond $0.5 \%$ strain. The change in anisotropy peaks at about $3000 \mathrm{Oe}$ at a strain between 1.2 and $1.5 \%$. Beyond this strain the anisotropy decreases rapidly, and the ferrimagnetic material becomes paramagnetic when the strain exceeds a value of about $2 \%$. There are slight differences between neon and helium implantation at high strain, but the behavior is remarkably consistent considering the great difference in mass and doping density. Typical data for $\mathrm{He}$ and $\mathrm{Ne}$ implantation are summarized in Fig. 1, which is a somewhat abbreviated presentation of Fig. 6 from Ref. 2.

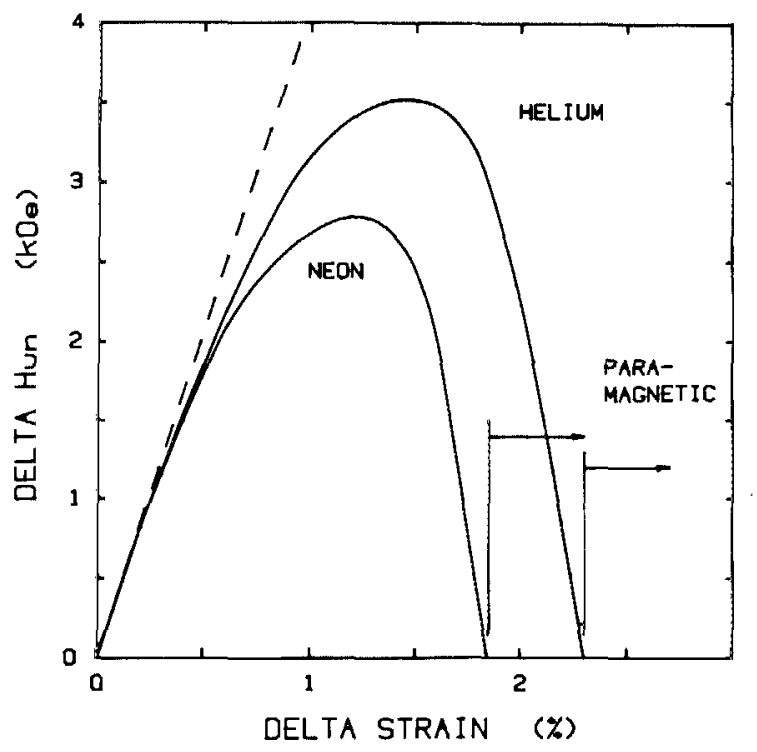

FIG. 1. Change in magnetic anisotropy with strain for neon and helium implantation (from Ref. 2). 
Hydrogen implantation exhibits a drastically different relation. ${ }^{2,7,8}$ This anomalous behavior has been known for several years, but a detailed description has been lacking. The present study was also aimed at quantifying and explaining this behavior.

\section{EXPERIMEENTAL DETAILS}

In this investigation, (111)-oriented Gd, Tm, Ga substituted yttrium iron garnet ( $\mathrm{Gd}, \mathrm{Tm}, \mathrm{Ga}: \mathrm{YIG}$ ) films about 3.5 mm square and $1 \mu$ thick were implanted with $\mathrm{H}_{2}{ }^{+}$at 120 $\mathrm{keV}$ with four doses ranging from about $3 \times 10^{15}$ to $4 \times 10^{16}$ ions $/ \mathrm{cm}^{2}$. Temperature during implantation was not measured, but the beam current was kept below $30 \mu \mathrm{A}$ to avoid excessive heating. Implantation time per sample ranged from 0.35 to $5.5 \mathrm{~h}$. Crystal strain conditions were obtained using double-crystal, Bragg case, $x$-ray rocking curves with a 50-keV CuK $\alpha \mathrm{x}$-ray beam. All samples were measured using (444) reflection, except for the lowest strain cases which used (888) reflection for greater sensitivity. Attempts to correlate the nominal dose with peak strain and peak anisotropy both with hydrogen and other implants ${ }^{5}$ have clearly shown that the nominal doses are not accurate; so the only reliable measure of implantation condition is provided by the peak strain perpendicular to the film plane which has been measured to an accuracy of about $2 \%$. The as-grown (Gd, Tm, Ga:YIG) film had a strain of about $0.07 \%$ with respect to the GGG substrate, and the peak increase in strain due to implantation was $0.35,0.60,1.50$, and $2.90 \%$ for the four coses, hereafter referred to as cases 1-4. The peak strain occurred at a depth of $3600 \AA$ for all doses. Magnetic characteristics of the implanted layer were found by analyzing ferromagnetic resonance (FMR) spectra obtained using a $10-\mathrm{GHz}$ cavity spectrometer. Data regarding implantation and magnetic properties of the unimplanted film are summarized in Table L.

After implantation, each film was annealed in air at successively higher temperatures from 200 to $600^{\circ} \mathrm{C}$, usually at $100^{\circ} \mathrm{C}$ intervals. The use of consecutive anneals of one

TABLE I. Physical and magnetic properties and implantation conditions of garnet films.

\begin{tabular}{lcl}
\hline $\begin{array}{l}\text { Nominal composition } \\
\left(\mathrm{Gd}_{0.84} \mathrm{Tm}_{1.17} \mathrm{Y}_{0.99}\right)\left(\mathrm{Fe}_{2}\right)\left(\mathrm{Ga}_{0.39} \mathrm{Fe}_{2.61}\right) \mathrm{O}_{12}\end{array}$ & \\
\hline $\begin{array}{l}\text { Nominal thickness } \\
4 \pi M\end{array}$ & 1 & $(\mu \mathrm{m})$ \\
$\gamma$ & 515 & $(\mathrm{G})$ \\
$A$ & $1.50 \times 10^{7}$ & $\left.(\mathrm{Oe})^{-1} \mathrm{sec}^{-1}\right)$ \\
$H_{1}$ & $2.9 \times 10^{-7}$ & $(\mathrm{erg} / \mathrm{cm})$ \\
$H_{k}$ & -165 & $(\mathrm{Oe})$ \\
Crystal strain (as grown) & 600 & $(\mathrm{Oe})$ \\
& 0.07 & $(\%)$ \\
\hline
\end{tabular}

Implantation $\mathrm{H}_{2}^{+}$at $120 \mathrm{keV}$

Case Nominal dose $\left(\mathrm{cm}^{-2}\right)$

Peak change in strain $(\%)$

\begin{tabular}{lrl}
\hline 1 & $3 \times 10^{15}$ & 0.35 \\
2 & $5 \times 10^{15}$ & 0.60 \\
3 & $20 \times 10^{15}$ & 1.52 \\
4 & $40 \times 10^{15}$ & 2.90 \\
\hline
\end{tabular}

sample, rather than single anneals of many samples, was justified by comparison of a consecutive 9-step (11-h total) annealing sequence of one garnet up to $350^{\circ} \mathrm{C}$ and a 2-step anneal $\left(1 \frac{1}{2} \mathrm{~h}\right.$ total) to the same temperature. Both samples showed the same anisotropy profiles (identical FMR spectra) for the two corresponding steps. The choice of $1-\mathrm{h}$ annealing time represents a compromise between shorter times which minimize laboratory work and longer times which more nearly reflect equilibrium conditions.

$\mathrm{X}$-ray rocking curves and FMR spectra were obtained after each anneal. For high-dose implantation there was a large change in magnetic properties below $200^{\circ} \mathrm{C}$, so for cases 3 and 4 annealing and FMR analysis were also carried out at $150^{\circ} \mathrm{C}$, but $\mathrm{X}$-ray rocking curves were not measured. Strain and magnetic profiles were obtained using the method described in Ref. 2. As in that reference, the anisotropy components are not readily separated with any accuracy, but the total which is determined accurately is given quantitatively by the change in local uniform perpendicular resonance field:

$$
\Delta H_{\mathrm{un}}=\Delta\left(\omega / \gamma+4 \pi M-H_{k}+2 / 3 H_{l}\right)
$$

If both perpendicular and parallel spectra are analyzed, additional information is obtained because in the parallel orientation the change in effective anisotropy is given approximately by a different relation:

$$
\left(\Delta H_{\text {un }}\right)_{\text {par }} \approx \Delta\left[\omega / \gamma-1 / 2\left(4 \pi M-H_{k}\right)+1 / 4 H_{l}\right] \text {. }
$$

Since very few surface modes are excited in parallel resonance, the parallel spectra are not suitable for determining profile shape. However, given the shape from the perpendicular spectrum, the parallel spectrum allows a partial separation of anisotropy components. In particular, as will be shown later, it allows a determination of the average value of $\gamma$ in the surface layer. Since this is an indirect measurement, a confirmation by a more direct method is desirable. For a small number of samples, the gyromagnetic ratio $\gamma$ was measured in both bulk and surface layers by measuring the shift in the FMR spectrum between two different frequencies. To avoid major change in microwave structure, two interchangeable cavities were used which resonated within the tuning range of a single V-153 klystron. This provided a 0.5 $G K z$ frequency shift and a corresponding shift in the spectra of about 200 Oe. Because of this small shift and the uncertainty introduced by temperature variation and sample alignment, the accuracy is limited to about $10 \%$, but this is sufficient to demonstrate or rule out any really significant chatiges in $\gamma$. Both parallel and perpendicular FMR were measured. Measurements were made before annealing for the lower doses, cases 1 and 2 , and for case 3 after $600^{\circ} \mathrm{C}$ annealing.

\section{RESUETS}

Strain profiles for all four doses as implanted and for annealing temperatures between 200 and $600{ }^{\circ} \mathrm{C}$ are shown in Fig. 2. For high doses, the strain decreases with annealing more rapidly relative to its initial value. This is better illustrated in Fig. 3 by plotting the peak strain versus annealing temperature for each case. Since the temperature during im- 

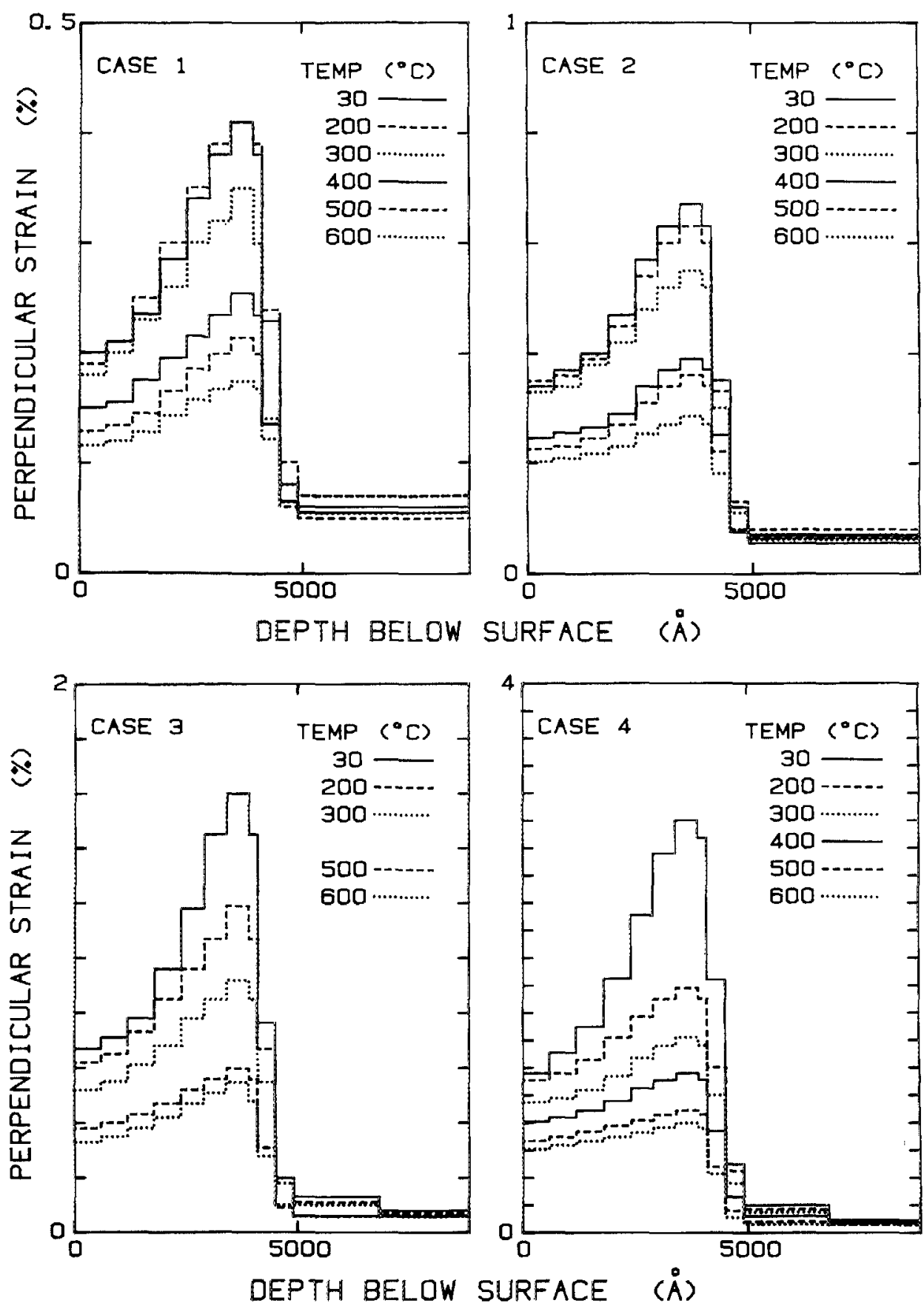

FIG. 2. Perpendicular strain profiles for hydrogen implantation with annealing temperature as parameter. Data labeled 30 indicates a room-temperature measurement of the as-implanted sample.

plantation is not known, data for as-implanted garnets are plotted at $30^{\circ} \mathrm{C}$ (room temperature). To facilitate comparison of data in this figure, all cases have been normalized to the same value at $500^{\circ} \mathrm{C}$. For the low-dose cases 1 and 2 , the decrease in strain with temperature is remarkably similar to that reported for neon and helium implantation (see Fig. 11 in Ref. 2). Cases 3 and 4 show the same similarity above $200^{\circ} \mathrm{C}$, but for the unannealed films there is a deviation that increases with dose. This deviation primarily involves an increase in strain in the region of expected maximum concentration of hydrogen atoms.

Another graphic picture of the effect is the comparison in Fig. 4 of the strain profile for each annealing sequence, normalizing the strain at the surface of the film. There will be a further justification for this normalization later when these profiles are compared with the anisotropy profiles. The fig- ure gives comparisons of the as-implanted films and after 300 and $500^{\circ} \mathrm{C}$ annealing. Here one can see a large deviation in the interior of the as-implanted film for high doses. This anomaly has greatly diminished at $200^{\circ} \mathrm{C}$ (not shown for clarity in the figure) and has almost disappeared at $300^{\circ} \mathrm{C}$.

For the highest dose case where the peak change in strain is $2.9 \%$, the garnet layer gives no indication of amorphousness. X-ray studies of a film with dose $8 \times 10^{16}$ ions/ $\mathrm{cm}^{2}$ indicate that a strain of $3.9 \%$ is required before all detectable diffraction disappears. At this strain, the standard deviation of the incoherent displacement of atoms is about $0.5 \AA$, in good agreement with the value $0.6 \AA$ found for $\mathrm{Ne}$ and $\mathrm{He}^{2}$

The FMR spectra for cases 1 and 2 are readity analyzed, and some of these data have already been presented in Fig. 10 of Ref. 2. However, there is a serious difficulty with the high- 


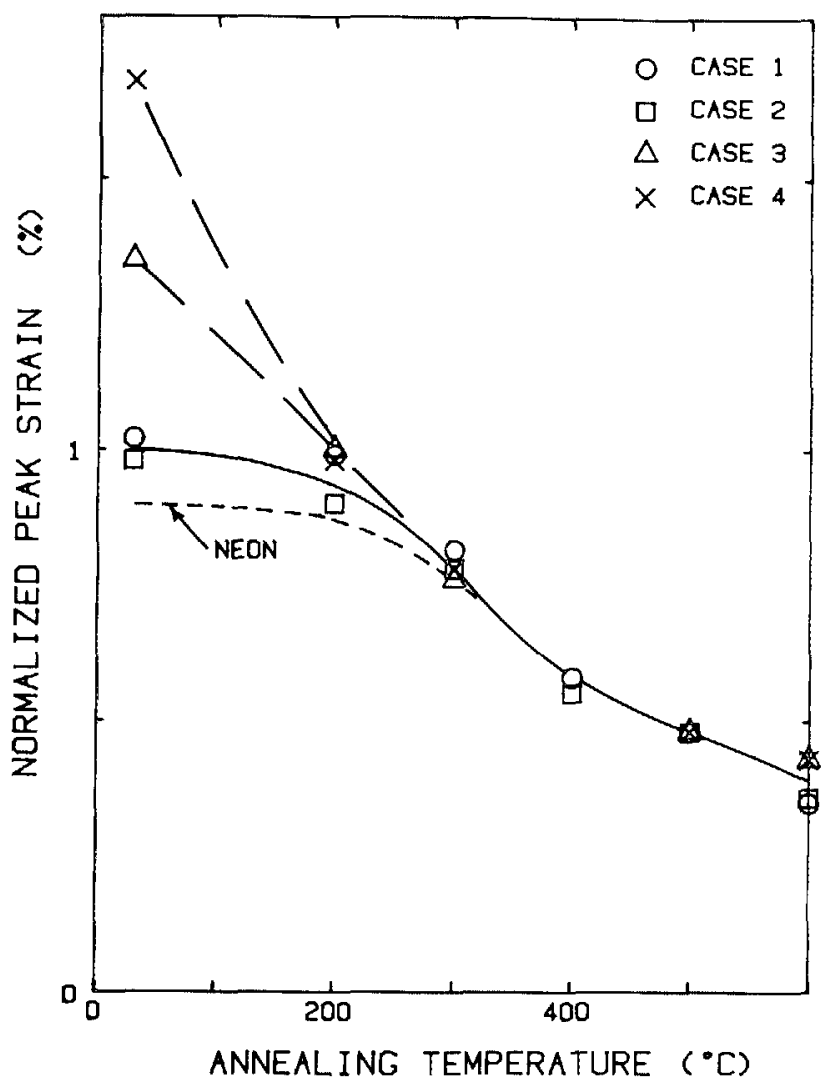

FIG. 3. Peak strain as a function of annealing temperature, normalized to a common value 0.48 at $500^{\circ} \mathrm{C}$. Data for neon is from Ref. 2 .

er doses. The change in anisotropy is so large that the field required for resonance of the highest surface mode exceeds the capability of the spectrometer magnet. Thus although the peak change in anisotropy cannot be measured, the maximum field of the magnet $(16 \mathrm{kG})$ sets a lower limit, so that the anisotropy is known to exceed $\Delta H_{\text {un }}=13000$ De. By comparing spectra of all four cases as a function of angle between perpendicular and parallel, it appears that the highest dose film, case 4, has become nonmagnetic (paramagnetic) in those regions that have highest strain at lower doses. The same may be true for case 3 , but in any case there are magnetic regions with a peak change of anisotropy in excess of $13 \mathrm{kG}$ as stated earlier.

At an annealing temperature of $150^{\circ} \mathrm{C}$, the highest dose (case 4) is still unmeasurable, but the peak surface mode for case 3 is found at 15800 Oe. This permits determination of the peak anisotropy to be $\Delta H_{\text {un }} \sim 12700$. For annealing temperatures of $200^{\circ} \mathrm{C}$ and above, the anisotropy has decreased in all four cases to values which can be easily measured on the spectrometer, except for a minor difficulty discussed below, and the FMR spectra uniquely determine the anisotropy profiles as has been discussed in Ref. 2 .

A special problem arises in the case of perpendicular FMR where the peak change in anisotropy $\left(\Delta H_{\mathrm{un}}\right)_{\max }$ is positive and exceeds the fundamental resonance field $\omega /$ $\gamma=4000$ Oe by a substantial amount. The highest surface mode is found as expected at a field just below

$$
\left(H_{\alpha}\right)_{\max }=\frac{\omega}{\gamma}+\left(\Delta H_{\text {un }}\right)_{\max },
$$

and higher order surface modes are found as the field is swept downward from this value. When the applied field falls below $\left(H_{a}\right)_{\max }$ by an amount equal to $\omega / \gamma$, the field is no longer sufficient to align the magnetization in the region of highest anisotropy change. The magnetic structure becomes divided into two regions, and the FMR calculation is no longer valid. Experimentally, one finds the expected spectrum in a field interval of roughly $(\omega / \gamma)$, and below this band sharp resonances no longer occur. In many cases the spectrum simply disappears until the field reaches the value which excites the main body mode of the unimplanted region. Since this mode is effectively pinned at the interface and does not involve excitation in the surface layer, this mode is not suppressed. An illustration of this structure is shown in Fig. 5 for case 3 after a $200^{\circ} \mathrm{C}$ anneal. Both experimental and calculated modes are shown. The upper four experimental modes lie in a band of about 4000 Oe and are fitted very well by the FMR calculation. The next lower mode is in substantial error, about $150 \mathrm{Oe}$ in location and a factor of 2 in amplitude. Moreover, the next six calculated surface modes are not observed in the experimental spectrum. A similar situation is found for case 4 after a $200^{\circ} \mathrm{C}$ anneal. In this case a flatter anisotropy profile now produces seven modes matching the experimental modes; an eighth mode is inaccurate, and ten additional modes predicted at lower fields are not observed experimentally. As is seen in Fig. 5, after annealing at $300^{\circ} \mathrm{C}$, the anisotropy change has fallen below $4000 \mathrm{Oe}$, and the full spectrum is observed.

Since the anisotropy changes due to other implant elements, such as $\mathrm{He}, \mathrm{O}$, and $\mathrm{Ne}$, saturate at $3000-3500 \mathrm{Oe}$, this effect is not observed in FMR spectroscopy at $10 \mathrm{GHz}$ for these implants. However, when a low-frequency spectrom-
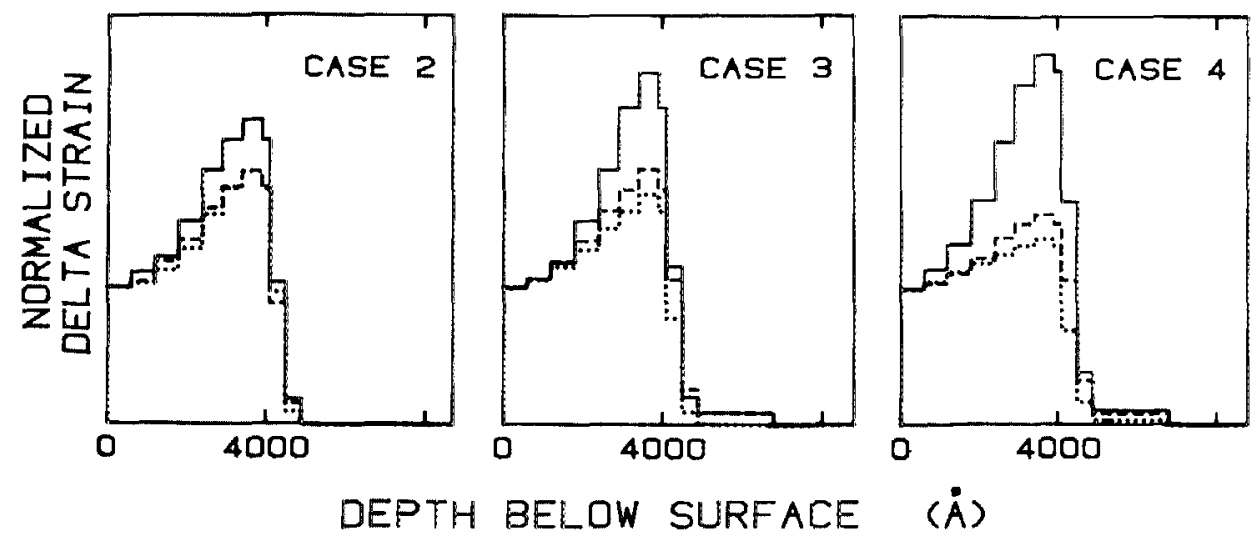

FIG. 4. Strain profiles normalized to equal values at the surface. Solid line, as implanted; dashed line after $300^{\circ} \mathrm{C}$ anneal; dotted line after $500^{\circ} \mathrm{C}$ anneal.

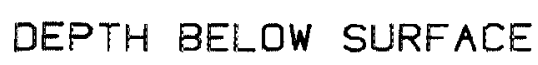

(A) 


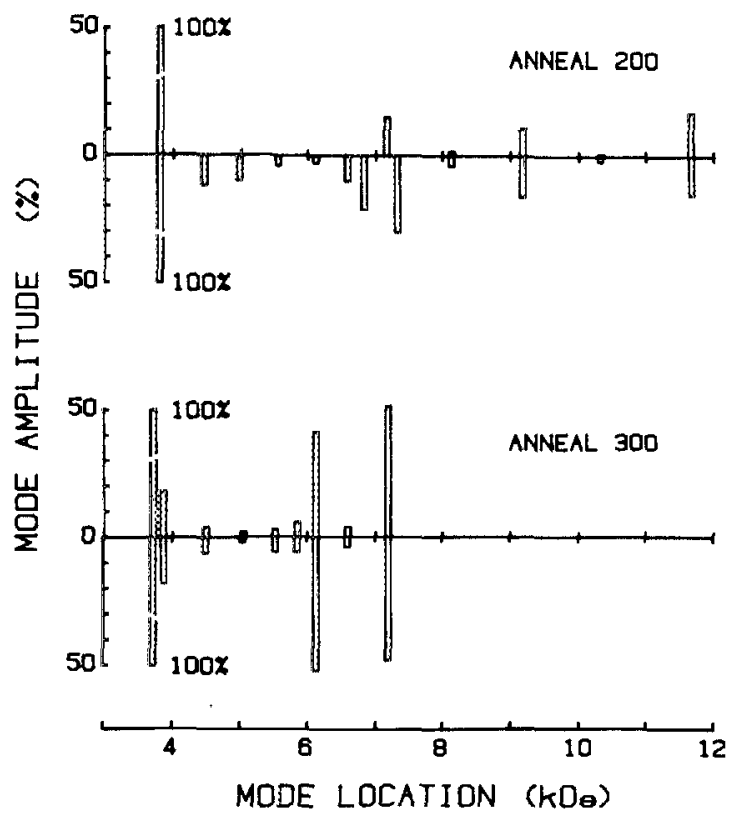

FIG. 5. Perpendicular FMR spectra for case 3 after annealing at 200 and $300^{\circ} \mathrm{C}$. The experimental spectrum is above axis, and the calculated spectrum below axis. The mode at far left is a principal body (unimplanted) mode, with amplitude defined as $100 \%$. All other modes shown are surface modes.

eter is employed, this same problem also occurs in these cases. $^{9}$

The anisotropy profiles obtained by fitting the FMR spectra are shown in Fig. 6 for as-implanted films and after annealing at 200,300 , and $500^{\circ} \mathrm{C}$. As with other implant species, these profiles are best compared with the strain profiles if they can be brought into concordance at low strains and low doses. This requires multiplication of the strain values by the factor $4200 \mathrm{Oe} / \%$, in very good agreement with the factor 4100 reported in Ref. 2 for Ne and He implantation.

The peak change in anisotropy has been plotted against peak strain in Fig. 7 with annealing temperature as parameter. All four cases are shown in the same figure. Figures 6 and 7 clearly show the very interesting result that the hydrogen anomaly is readily removed by annealing. This has been previously reported by Hirko and $\mathrm{Ju},{ }^{7}$ but not with the detail shown here. The peak anisotropy before annealing exceeds that which would be observed at the same strain with helium or neon implantation. For low-dose cases 1 and 2, the increase is a factor of 1.6 and 2.1 , respectivelly, and for case 3 the value is unknown but lies above $\Delta H_{\mathrm{un}}=13000$ Oe. All four cases approach the neon-helium implantation line (Fig. 1) as the annealing temperature increases, and they have effectively converged on this line for temperatures above $400^{\circ} \mathrm{C}$.

As stated earlier, analysis of both perpendicular and parallel spectra gives additional information about magnetic parameters, allowing a partial separation of $\left(H_{k}-4 \pi M\right)$ from $H_{1}$ and $\omega / \gamma$. However, it is not possible to directly separate changes in $\omega / \gamma$ from changes in $H_{1}$. For example, in case 2 as implanted, the profile of $\Delta H_{\mathrm{un}}$ required to match the perpendicular FMR spectrum reaches a peak value of $4200 \mathrm{Oe}$ as shown in Fig. 6. If no change is assumed in $H_{1}$ or
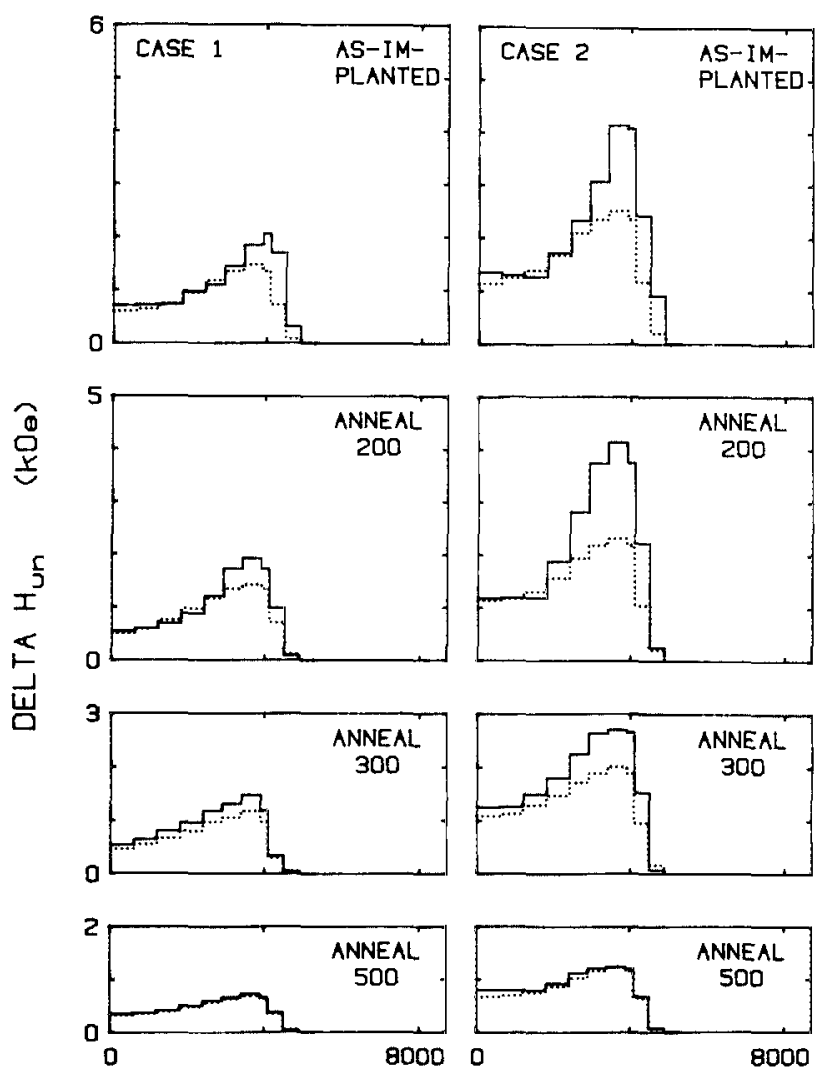

DEPTH BELOW SURFACE (A)
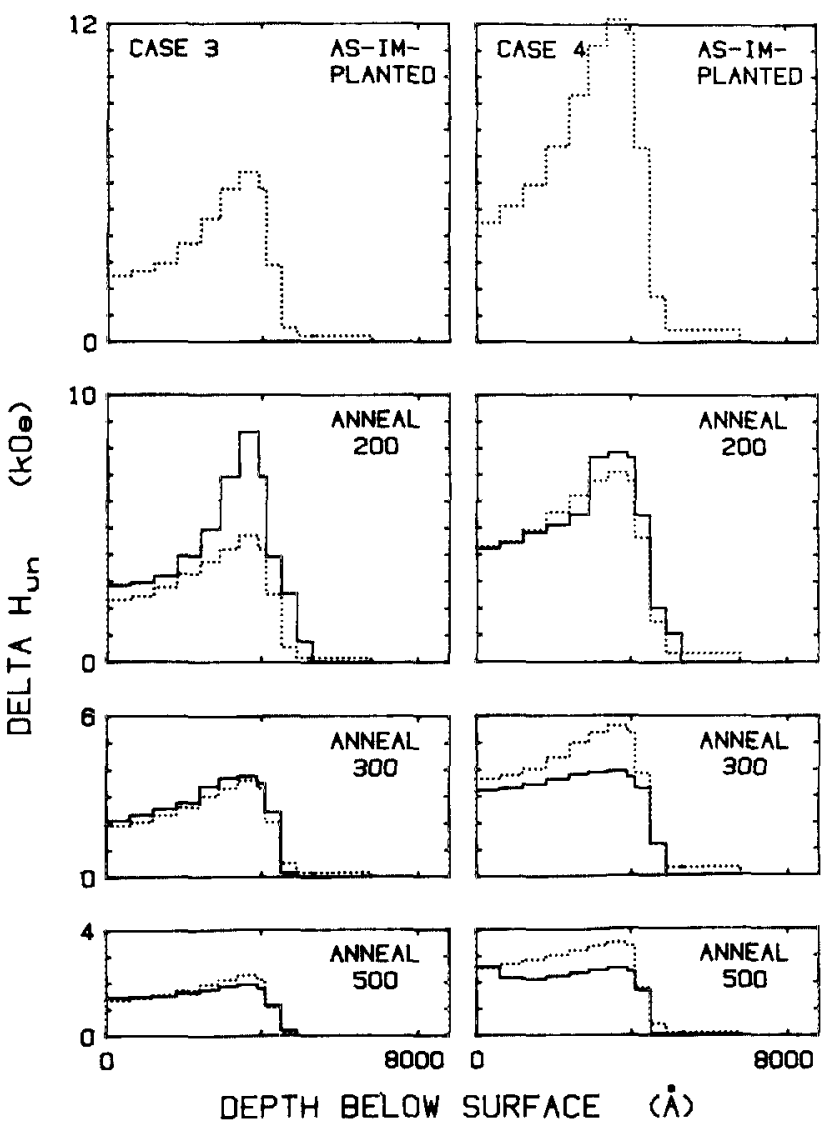

FIG. 6. Comparison of profiles of magnetic anisotropy (solid line) and strain (dotted line) for various annealing temperatures up to $500^{\circ} \mathrm{C}$. Strain has been multiplied by 4200 for ease of comparison. 


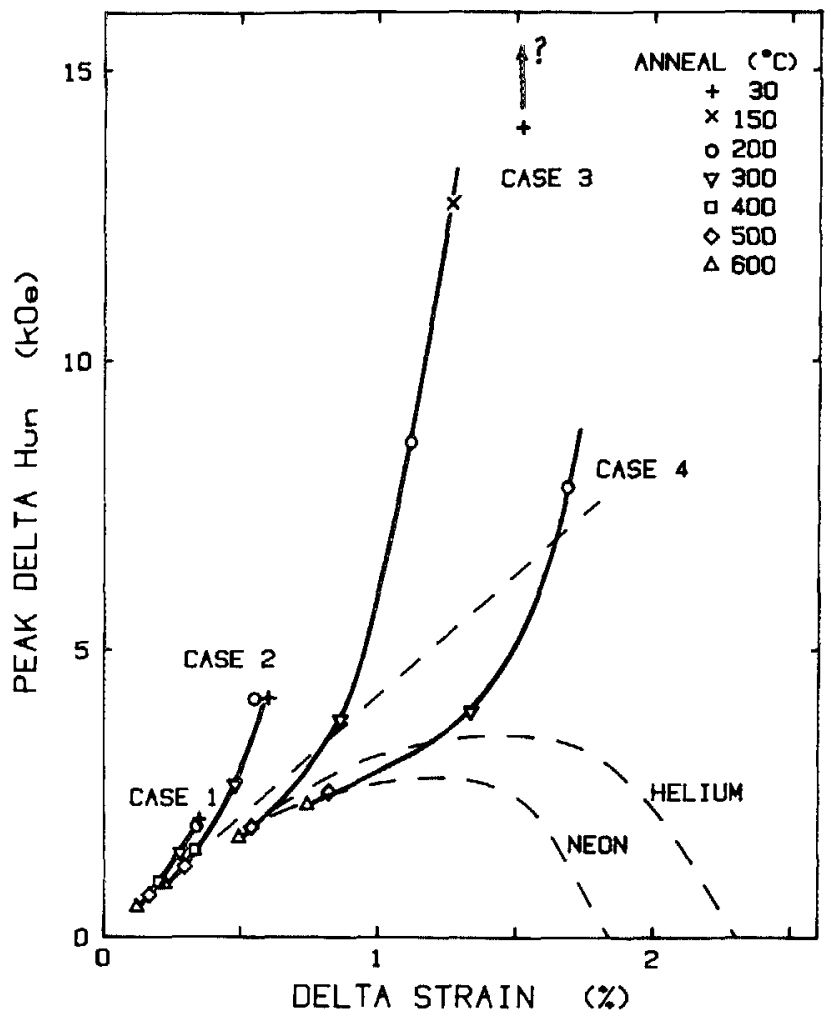

FIG. 7. Peak change in anisotropy vs. peak change in strain with annealing temperature as parameter. For ease of comparison with other implantation elements, Fig. 1 is overlaid in dotted line. Data labeled 30 indicates roomtemperature measurement of as-implanted sample.

$\omega / \gamma$, the parallel spectrum cannot be fitted, and the location of the principal surface mode in parallel resonance is in error by $91 \mathrm{Oe}$. This can be corrected by assuming a 370-Oe change in $H_{1}$ from - 165 Oe in the unimplanted region to a peak value of +205 Oe in the implanted layer, by a $3 \%$ decrease in $\gamma$ in the implanted layer, or by a combination involving smaller changes in both.

This can be partly resolved by studying the asymmetry of the FMR spectrum as a function of angle, for rotation about an $\langle 011\rangle$ axis. Although accurate characterization is impossible, the observed asymmetry still requires a negative value of $H_{1}$ in the implanted layer and sets a rough limit in the peak value of $H_{1}$ to the range $-140<H_{1}<-80$, which indicates a change about five times smaller than that required above. In other words, in this case the change in $(\omega / \gamma)$ must dominate and requires a decrease in $\gamma$ of about $2.5 \%$. Similar information is given in Table II for all cases for which parallel FMR spectra were obtained. It should be recognized that the change in $\gamma$ obtained from this analysis can

TABLE II. Peak change in $\gamma$ in implanted layer in percent. Values uncertain to $0.5 \%$ due to uncertainty in surface crystalline anisotropy. Parallel spectra were not measured where data are omitted.

\begin{tabular}{cccccccc}
\hline \hline & \multicolumn{7}{c}{ Annealing temperature $[\mathrm{C})$} \\
\cline { 2 - 8 } Case & 30 & 200 & 300 & 400 & 500 & 600 & 700 \\
\hline 1 & -2.0 & -1.5 & -1 & -0.3 & +1.1 & +0.5 & $\ldots$ \\
2 & -2.5 & -1.5 & -0.5 & -0.5 & -0.5 & +0.5 & $\cdots$ \\
3 & $\cdots$ & $\cdots$ & $\cdots$ & $\cdots$ & $\cdots$ & +1.5 & +1.8 \\
4 & $\cdots$ & $\cdots$ & $\cdots$ & $\cdots$ & $\cdots$ & $\cdots$ & +2.5 \\
\hline \hline
\end{tabular}

be in error as much as $0.5 \%$ because of uncertainty in the value of $H_{1}$. Although this error is not much smaller than the changes observed, the data have good consistency and show that the change in $\gamma$ is quite negligible in all cases.

The variations observed here are far smaller than the variation reported by $\mathrm{Krafft}$ and $\mathrm{Kryder}^{10}$ for deuterium implantation. For this reason independent verification for a few cases was carried out using the same method as in Ref. 10, namely the shift in spectrum with frequency. The accuracy of 5 or $10 \%$ in this determination is limited by experimental difficulties and the small shift in frequency used. However, for the three cases measured, cases 1 and 2 as implanted and case 3 at a $600^{\circ} \mathrm{C}$ anneal, all values differed from Table II by amounts less than the probable error.

The magnetic parameters $4 \pi M$ and $A$ in the implanted layer cannot be individually determined with a precision even approaching that for the anisotropy. However, the ratio $(A / M)$ is a fundamental parameter in the differential equation describing the resonance, and its average value, and to a certain extent even its profile, must be set correctly to secure a fit to the resonance spectrum. The average values of $A / M$ for all four cases and all annealing temperatures are shown in Fig. 8. Data for cases 3 and 4 at $200^{\circ} \mathrm{C}$ are only approximate since the entire spectrum is unavailable experimentally, as discussed elsewhere in this report. Other data are believed to have an accuracy of about $10 \%$.

\section{DISCUSSION}

The principal results of this investigation are the quantitative measurement of the large anisotropy shift produced by hydrogen implantation and a detailed description of its disappearance with annealing. It is unfortunate that conventional FMR spectrometers cannot measure reliably the anisotropy shift that occurs when the preanneal implantation

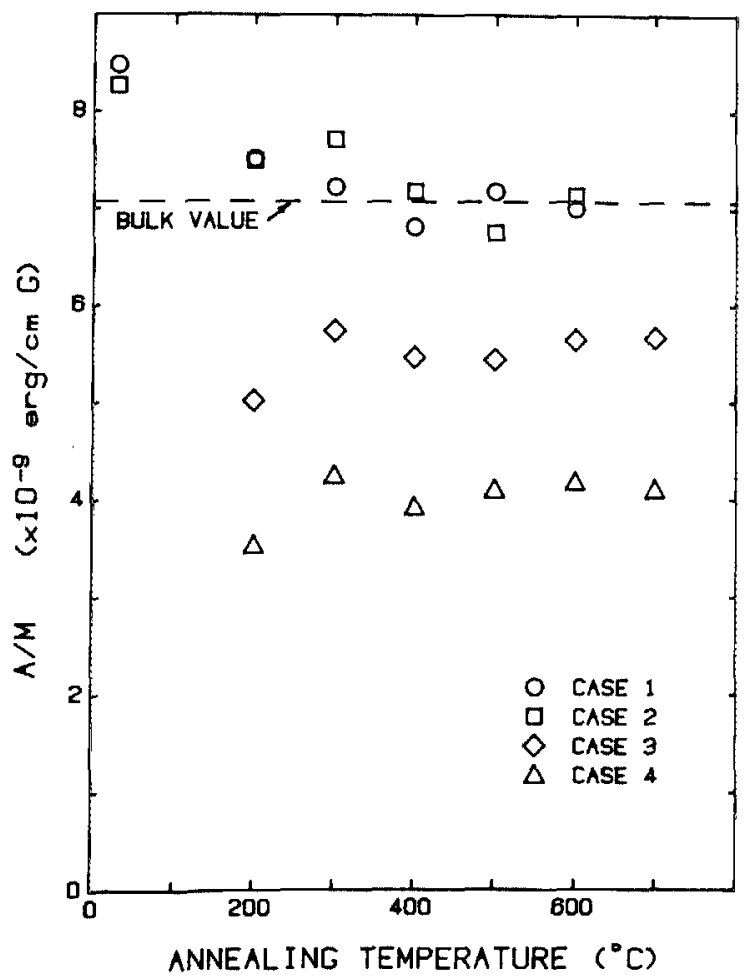

FIG. 8. Average value of $A / M$ in implanted layer as a function of annealing temperature. 
strain exceeds $1 \%$, but it is at least clear that anisotropies in excess of $H_{k}=-13000$ Oe are generated. Upon annealing beyond $300^{\circ} \mathrm{C}$, the anisotropies decrease remarkably and become commensurate with those obtained at the same strain with other implants such as $\mathrm{He}, \mathrm{O}$, and $\mathrm{Ne}^{5,10}$ It is plausible to speculate that the excess anisotropy shown in Figs. 6 and 7 is due to the presence of hydrogen which is desorbed upon annealing at $300-400^{\circ} \mathrm{C}$. This further suggests that the anisotropy can be regarded as made up of two components, one due simply to the crystal strain and the other due to the presence of hydrogen atoms. These will be discussed separately.

As shown in Fig. 7, for $\mathrm{Ne}$ and $\mathrm{He}$ implantation $\Delta H_{\text {un }}$ saturates and decreases to zero as the material becomes paramagnetic at a strain around $2 \%$. We speculate that this is due to damage, rather than strain. As incoherent atomic displacements increase, the symmetry of the unit cell changes from cubic to orthorhombic to nearly spherically symmetric at the amorphous level. The destruction of uniaxial symmetry is reflected in the final destruction of the uniaxial $\Delta H_{u n}$. Just below the paramagnetic level the material resonates at a field equal to $\omega / \gamma$. Since damage also occurs with hydrogen implantation, one expects an eventual saturation and decrease in $\Delta H_{\mathrm{un}}$. The saturation appears to be masked by the large contribution associated with the presence of hydrogen. Since hydrogen is desorbed at or below $400^{\circ} \mathrm{C}$, one should see evidence of the saturation after annealing, if the residual strain and damage are sufficiently large. This appears to be confirmed in Fig. 7 for the highest dose case.

The anisotropy component due to the presence of hydrogen is likely to depend on the chemical nature of this atom, but one cannot rule out $a$ priori the possibility that it simply depends on the density of foreign atoms. There is little or no experimental data with other implants to resolve this question. Figure 7 demonstrates that the effect is nonlinear and in any case requires a dose in the range $(2-3) \times 10^{15}$ ions of $\mathrm{H}_{2}$ or $4-6 \times 10^{15}$ protons $/ \mathrm{cm}^{2}$ to obtain a significant anisotropy component. Implantation at this dose with most other elements renders the implanted layer nonmagnetic and even amorphous. Even a light element like He saturates at a dose of $6 \times 10^{15} \mathrm{~cm}^{-2}$. This same dose of $\mathrm{H}$ gives an excess anisotropy of about $700 \mathrm{Oe}$ in Fig. 7. Since the saturation curve for helium lies above that for neon (and presumably other heavy atoms) by about this amount, it cannot be ruled out that this difference is due to the presence of helium atoms. However, it seems more likely that the saturation, being due to damage rather than strain, occurs at higher strain for helium because the damage as measured by the random displacement $U$ is considerably smaller for the lighter element. Annealing of the helium implants cannot be used to distinguish these two effects for three reasons. The temperature required for desorption is so high that little residual strain will be left; the excess anisotropy if it exists is small; and finally high-dose helium implants are damaged by annealing, apparently due to the formation of large bubblelike defects.

However, further evidence of the effect may be available using deuterium, which is chemically similar to hydrogen. Implantation with deuterium has been studied by
TABLE III. Comparison of $\Delta H_{u n} / \Delta$ strain for hydrogen and deuterium implantation before annealing. (Data for deuterium from Ref. 10.)

\begin{tabular}{cccc}
\hline \hline Implant Ion & $\begin{array}{c}\text { Nominal Dose } \\
\left(\mathrm{cm}^{-2}\right)\end{array}$ & $\begin{array}{c}\Delta \text { Strain } \\
(\%)\end{array}$ & $\begin{array}{c}\Delta H_{\mathrm{un}} / \Delta \text { Strain } \\
(\mathrm{Cc} / \%)\end{array}$ \\
\hline $\mathrm{H}_{2}^{+}$ & $5 \times 10^{15}$ & 0.6 & 7400 \\
$\mathrm{H}_{2}^{+}$ & $20 \times 10^{15}$ & 1.5 & $>10000$ \\
$\mathrm{D}_{2}^{+}$ & $12 \times 10^{15}$ & 1.5 & $\sim 3500$ \\
\hline
\end{tabular}

Krafft and Kryder. ${ }^{10,11}$ Since the anisotropy change depends on garnet composition, it is fortunate that they include oxygen implantation data which can be used for calibration purposes. It is noteworthy that the initial slope $\Delta H_{k} / \Delta \epsilon=4000$ $\mathrm{Oe} / \%$ for oxygen is nearly identical to that obtained for $\mathrm{He}$ and $\mathrm{Ne},{ }^{5}$ even though different garnets were used. The data for deuterium are given in Fig. 1 of Ref. 10 and consist of a single dose in three samples giving a strain of $1.5 \%$ and an anisotropy change of about $5000 \mathrm{Oe}$. Table III compares this with the results for hydrogen. Although the actual doses are uncertain, the relative values seem consistent ( $D$ with twice the mass should produce more strain for a given dose). Anisotropy change for a given dose of deuterium is not only much smaller than that for the same strain with hydrogen but also much smaller than obtained with a smaller strain but the same density of hydrogen. The value from Table 1 HI if plotted on Fig. 7 lies somewhat above the lines for neon or helium but far below the corresponding point for hydrogen (symbol + for case 3 ). The deviation from the helium line may represent the contribution due to the presence of deuterium, but if so, the increase is surprisingly small compared with that due to hydrogen. It will not be possible to resolve the apparent discrepancy without further experimental work at other doses.

The gyromagnetic ratio is relatively unaffected by hydrogen implantation at the doses reported here. The slight variations shown in Table $\mathrm{L}$ are not much larger than the uncertainty of measurement; so the shift may not be real. In any case it is much smaller than the $30 \%$ decrease reported by Krafft and Kryder for deuterium implantation.

The difference in $A / M$ between the two lowest doses and two highest doses shown in Fig. 7 merits discussion. For cases 1 and 2, the variation with dose is small and appears to disappear with annealing beyond $300^{\circ} \mathrm{C}$. For the high-close cases where extreme changes are observed in the magnetic parameters, the value of $A / M$ is irrevocably altered by the implantation and subsequent annealing. In the highest dose case, $A / M$ is lowered by a factor of nearly two.

'V. S. Speriosu, J. Appl. Phys. 52, 6094 (1981).

${ }^{2}$ V. S. Speriosu and C. H. Wilts, J. Appl. Phys. 54, 3325 (1983).

${ }^{3} \mathrm{~J}$. C. North and R. Wolfe, in Ion-Implantation in Semiconductors and Other Materials, edited by B. L. Crowder (Plenum, New York, 1973).

${ }^{4}$ B. E. MacNeal and V. S. Speriosu, J. Appl. Phys. 52, 3935 (1981).

'H. Awano, V. S. Speriosu, and C. H. Wilts, J. Appl. Phys. 55, 3043 (1984).

${ }^{6}$ Y. Sugita, T. Takeuchi, and N. Ohta (unpublished).

${ }^{7}$ R. Hirko and K. Ju, IEEE Trans. Magn. MAG-16, 958 (1980).

${ }^{8}$ Y. Satoh, M. Ohashi, T. Miyashita, and K. Komenou, J. Appl. Phys. 53, 3740 (1982).

${ }^{9} \mathrm{C}$. S. Kraff (private communication).

${ }^{10} \mathrm{C}$. S. Krafft and M. H. Kryder, IEEE Trans. Magn. MAG-20, 1111 (1984).

"C. S. Kraff and M. H. Kryder, J. Appl. Phys. 55, 2557 (1984). 